\title{
The anti-ageing molecule sirt1 mediates beneficial effects of cardiac rehabilitation
}

\author{
Giusy Russomanno ${ }^{1 \dagger}$, Graziamaria Corbi ${ }^{2 \dagger}$, Valentina Manzo ${ }^{1}$, Nicola Ferrara ${ }^{3,4}$, Giuseppe Rengo ${ }^{3,4}$, \\ Annibale A. Puca ${ }^{1,5}$, Salvatore Latte ${ }^{6}$, Albino Carrizzo ${ }^{7}$, Maria Consiglia Calabrese ${ }^{1}$, Ramaroson Andriantsitohaina ${ }^{8}$, \\ Walter Filippelli ${ }^{9}$, Carmine Vecchione ${ }^{1,7}$, Amelia Filippelli ${ }^{1 *}$ and Valeria Conti ${ }^{1}$
}

\begin{abstract}
Background: An exercise-based Cardiac Rehabilitation Programme (CRP) is established as adjuvant therapy in heart failure (HF), nevertheless it is underutilized, especially in the elderly. While the functional and hemodynamic effects of CRP are well known, its underlying molecular mechanisms have not been fully clarified. The present study aims to evaluate the effects of a well-structured 4-week CRP in patients with stable HF from a molecular point of view.

Results: A prospective longitudinal observational study was conducted on patients consecutively admitted to cardiac rehabilitation. In fifty elderly HF patients with preserved ejection fraction (HFpEF), levels of sirtuin 1 (Sirt1) in peripheral blood mononuclear cells (PBMCs) and of its targets, the antioxidants catalase (Cat) and superoxide dismutase (SOD) in serum were measured before (Patients, P) and at the end of the CRP (Rehabilitated Patients, RP), showing a rise of their activities after rehabilitation.

Endothelial cells (ECs) were conditioned with serum from P and RP, and oxidative stress was induced using hydrogen peroxide. An increase of Sirt1 and Cat activity was detected in RP-conditioned ECs in both the absence and presence of oxidative stress, together with a decrease of senescence, an effect not observed during Sirt1 and Cat inhibition.

Conclusions: In addition to the improvement in functional and hemodynamic parameters, a supervised exercisebased CRP increases Sirt1 activity and stimulates a systemic antioxidant defence in elderly HFpEF patients.

Moreover, CRP produces antioxidant and anti-senescent effects in human endothelial cells mediated, at least in part, by Sirt1 and its target Cat.
\end{abstract}

Keywords: Heart failure, Rehabilitation, Sirtuin, Catalase, Oxidative stress, Endothelium

\section{Background}

Despite recent advances in clinical/diagnostic tools and therapies, the incidence and prevalence of Heart Failure (HF) show a steady increase [1]. A cardiac rehabilitation programme (CRP) based on exercise training, has been recognized as a fundamental component in the continuum of care for patients with HF. Meta-analyses of randomized controlled trials on CRPs have demonstrated a significant reduction of all-cause mortality, with lower rates of re-infarction and cardiac mortality [2].

\footnotetext{
* Correspondence: afilippelli@unisa.it

${ }^{\dagger}$ Equal contributors

'Department of Medicine, Surgery and Dentistry, University of Salerno, Via S.

Allende 43, 84081 Baronissi, Italy

Full list of author information is available at the end of the article
}

However, these studies included very few elderly or high-risk patients, and exercise is rarely viewed as a necessary prescription for these patients because they have more barriers to participation in exercise training [3].

In HF patients, exercise was shown to be associated with significant improvement in functional and hemodynamic parameters [4-7], nevertheless there are few data explaining the molecular mechanisms underlying exercise-based CRP.

It has been established both in humans and in animal models that exercise training can stimulate the natural antioxidant defences thereby contrasting reactive oxygen species (ROS) accumulation $[8,9]$.

The $\mathrm{NAD}^{+}$-dependent deacetylase sirtuin 1 (Sirt1) is now recognized as a mediator of the response to 
oxidative stress and endothelial dysfunction, phenomena both correlated with endothelial cell pathophysiology and Cardiovascular Diseases (CVDs), including HF [10]. Evidence about the protective role of Sirt1 in vascular biology has indicated Sirt1 as a possible target in preventing CVDs and other diseases [11, 12]. Indeed, Sirt1 plays a crucial role in both cellular senescence and ageing, and it was recognized as modulator of the oxidative stress response by inducing the expression of antioxidant enzymes such as superoxide dismutase (SOD) and catalase (Cat) [13, 14]. Recently, Lu et al. [15] showed that in advanced HF, low Sirt1 expression in ageing might be a significant contributing factor in the downregulation of antioxidants and upregulation of oxidative stress and apoptosis. We previously showed that moderate exercise promoted Sirt1 activity in rats and induced increasing SOD and Cat expression. Notably, in aged sedentary rats there was lower levels of Cat comparing to young rats and exercise led to a complete recover of such antioxidant enzyme [16].

Cat, a molecular target of Sirt1, represents a primary safeguard of the antioxidant system [17], and recent studies have suggested that this enzyme might play an important role in the pathophysiology of $\operatorname{HF}[18,19]$.

In humans, exercise training improves cardiovascular function and endothelial homeostasis, although the benefit achieved varies widely depending on the type and duration of exercise [20,21].

In the present study, we aim at investigating the molecular changes possibly induced by a 4-week CRP on Sirt1 activity in peripheral blood mononuclear cells (PBMCs) and antioxidant status in serum of patients with stable HF. Moreover, we looked at changes induced by the conditioning of human endothelial cells, exposed or not to oxidative stress induced by $\mathrm{H}_{2} \mathrm{O}_{2}$, with serum isolated from patients before and at the end of the CRP.

\section{Results}

\section{Heart failure elderly patient recruitment and} characterisation

Fifty-three consecutive patients affected by HF were recruited from the Cardiac Rehabilitation Unit. All patients completed the CRP. As only three patients were women, they were excluded from the analysis. Therefore, the final study population consisted of 50 elderly male patients (mean age 68.6 \pm 6.3 years). None of the patients had experienced a myocardial infarction (MI) in the 12 months preceding the study. All patients were in clinically stable condition, and classified as in NYHA II and III class with a preserved Ejection Fraction (EF) [10 patients with HF mid-range EF; 40 with HF preserved EF]. All definitions were based on the ESC and ACCF/AHA criteria, in which the term "stable" defines treated patients with symptoms and signs that have remained generally unchanged for at least 1 month [22].

The clinical and demographic features of the study population are listed in Table 1. Information on comorbidities and concomitant medications were gathered from all patients. No racial/ethnic-based differences were present. At baseline, no differences in medical therapy were found, and no changes occurred during the study period.

Data are expressed as mean (SD) or number of subjects (\%). BMI, Body Mass Index; SBP, Systolic Blood Pressure; DBP, Diastolic Blood Pressure; HR, Heart Rate; bpm, beat/minutes; CAD, Coronary Artery Disease; PTCA, Percutaneous Transluminal Coronary Angioplasty; CABG, Coronary Artery Bypass Graft; COPD, Chronic Obstructive Pulmonary Disease; ARBs, Angiotensin II Receptor Blockers.

Table 1 Study population characteristics and medication use

\begin{tabular}{|c|c|c|c|}
\hline Age (y.o.), mean (SD) & $68.6(6.3)$ & Medications, n (\%) & \\
\hline Gender (M/F) & $50 / 0$ & $\beta$-blockers & $\begin{array}{l}46 \\
(92)\end{array}$ \\
\hline $\mathrm{BMI}\left(\mathrm{kg} / \mathrm{m}^{2}\right)$, mean $(\mathrm{SD})$ & $\begin{array}{l}28.03 \\
(3.17)\end{array}$ & ACE-inhibitors & $\begin{array}{l}29 \\
(58)\end{array}$ \\
\hline $\mathrm{SBP}(\mathrm{mmHg})$, mean (SD) & $122(6)$ & ARBs & $9(18)$ \\
\hline DBP (mmHg), mean (SD) & $80(9)$ & Diuretics & $8(16)$ \\
\hline HR (bpm), mean (SD) & $84(8)$ & Nitrates & $6(12)$ \\
\hline CAD, $n(\%)$ & $49(98)$ & $\mathrm{Ca}^{2+}$-antagonists & $4(8)$ \\
\hline ischemic & $47(94)$ & a-antagonists & $2(4)$ \\
\hline hypertrophic & $1(2)$ & Aspirin & $\begin{array}{l}46 \\
(92)\end{array}$ \\
\hline dilatative & $1(2)$ & Anticoagulants & $\begin{array}{l}31 \\
(62)\end{array}$ \\
\hline PTCA, $n(\%)$ & $33(66)$ & Other cardiac drugs & $3(6)$ \\
\hline $\mathrm{CABG}, n(\%)$ & $9(18)$ & Antiarrhythmics & $2(4)$ \\
\hline Valvular substitution, $n$ (\%) & $1(2)$ & Statins & $\begin{array}{l}49 \\
(98)\end{array}$ \\
\hline Smoking, $n(\%)$ & $35(70)$ & $\begin{array}{l}\text { Gastro-protective } \\
\text { drugs }\end{array}$ & $\begin{array}{l}44 \\
(88)\end{array}$ \\
\hline Familiarity, $n$ (\%) & $15(30)$ & Polyunsaturated fats & $\begin{array}{l}13 \\
(26)\end{array}$ \\
\hline Hypertension, n (\%) & $29(58)$ & Oral hypoglycaemics & $9(18)$ \\
\hline Dyslipidaemia, n (\%) & $28(56)$ & Insulin & $5(10)$ \\
\hline Diabetes, $n(\%)$ & $13(26)$ & & \\
\hline COPD, $n(\%)$ & $6(12)$ & & \\
\hline Obesity, $n(\%)$ & $4(8)$ & & \\
\hline $\begin{array}{l}\text { Peripheral Artery Disease, } n \\
(\%)\end{array}$ & $2(4)$ & & \\
\hline Arrhythmias, $n(\%)$ & $2(4)$ & & \\
\hline Distyroidism, n (\%) & $1(2)$ & & \\
\hline Other diseases, $n$ (\%) & $1(2)$ & & \\
\hline
\end{tabular}


Biochemical, echocardiographic and cardiopulmonary stress test features of patients before $(\mathrm{P})$ and at the end of the CRP (RP) are shown in Table 2. A CRP significantly reduced cholesterol and increased creatinine levels (both $P<0.0001$ ).

Cardiopulmonary stress test revealed a reduction in maximum systolic blood pressure $(P=0.002)$, and increased maximum heart rate $(P=0.034)$, rate-pressure product $(P<0.0001)$, test duration $(P<0.0001)$, and VO2 $\max (P<0.0001)$ with consequent significantly higher exercise tolerance, one of the most crucial target in the HF treatment, after CRP.

\section{CRP-induced changes in antioxidant capacity in heart failure elderly patients}

The activity of Sirt1 and of its molecular targets, Cat and SOD before and at the end of the CRP were evaluated.

The CRP enhanced Sirt1 activity measured in PBMCs from patients (RP vs P, $P=0.02$ ) (Fig. 1, Panel a). Likewise, Cat and SOD activities measured in serum were greater in RP than in $\mathrm{P}(P<0.005$ and $P<0.05$, respectively) (Fig. 1, Panels b and c).

\section{Sirt1, Cat and SOD activities in endothelial cells} conditioned with patients' sera

To investigate the possible role of Sirt1, Cat and SOD in modulating the beneficial effects of the CRP, an in

Table 2 Changes in biochemical, echocardiographic and cardiopulmonary stress test parameters induced by CRP

\begin{tabular}{llll}
\hline & $P$ & $R P$ & $P$ value \\
\hline Biochemistry & & & \\
Cholesterol (mmol/L) & $157.61 \pm 37.11$ & $150.67 \pm 30.37$ & $<0.0001$ \\
Creatininemia (mmol/L) & $0.94 \pm 0.24$ & $0.97 \pm 0.20$ & $<0.0001$ \\
Hemoglobin (g/dL) & $13.58 \pm 1.35$ & $13.42 \pm 0.53$ & 0.494 \\
Echocardiographic parameters & & & \\
EF (\%) & $53.33 \pm 8.97$ & $55.10 \pm 6.79$ & 0.011 \\
LVEDD (mm) & $51.37 \pm 3.77$ & $51.31 \pm 3.13$ & 0.804 \\
Cardiopulmonary stress test & & & \\
SBP max (mmHg) & $169.02 \pm 15.54$ & $165.51 \pm 19.29$ & 0.002 \\
DBP max (mmHg) & $81.46 \pm 5.62$ & $80.64 \pm 5.15$ & 0.077 \\
HR max (bpm) & $117.56 \pm 21.55$ & $123.13 \pm 13.20$ & 0.034 \\
Rate-pressure product & $19892.69 \pm$ & $20261.54 \pm$ & $<0.0001$ \\
(mmHg x bpm) & 3841.64 & 3743.32 & \\
Test duration (sec) & $359.41 \pm$ & $451.89 \pm$ & $<0.0001$ \\
& 112.57 & 109.78 & \\
VO2 max (ml/kg/min) & $20.30 \pm 4.61$ & $24.51 \pm 5.81$ & $<0.0001$
\end{tabular}

P, HF patients pre-CRP; RP, the same patients post-CRP. Data are expressed as mean $\pm S D$. EF, Ejection Fraction; $L V E D D$, Left Ventricle End Diastolic Diameter; $S B P$, Systolic Blood Pressure; DBP, Diastolic Blood Pressure; $H R$, Heart Rate; $\mathrm{bpm}$, beat/minutes. A $P$ value $<0.05$ was considered significant

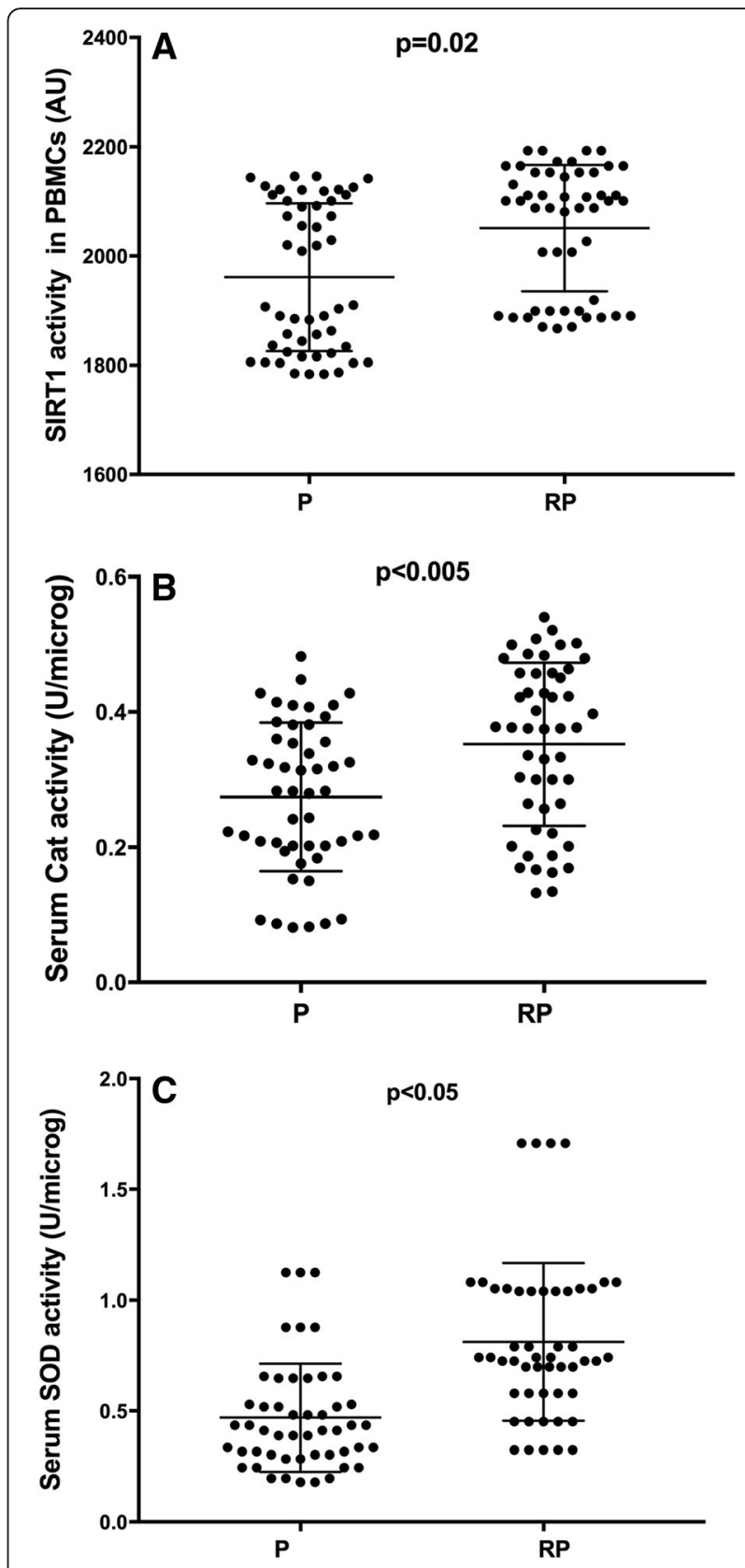

Fig. 1 CRP increased Sirt1 activity in PBMCs with a concomitant rise of antioxidants in sera. Sirtuin 1 (Sirt1) activity (a) was determined in the nuclei extracted from PBMCs from patients before (P) and after 4 weeks of a cardiac rehabilitation programme (CRP). Catalase (Cat) activity (b) and superoxide dismutase (SOD) activity (c) were determined in serum samples

vivo-in vitro model was set up by conditioning human endothelial cells (ECs) with sera from patients at time 0 (Patient serum-conditioned ECs, P-ECs) and at the end of the CRP (Rehabilitated Patient serumconditioned ECs, RP-ECs). Moreover, the antioxidant response in such conditioned cells was evaluated after the induction of stress using $\mathrm{H}_{2} \mathrm{O}_{2}$. 
Sirt1 and Cat activities were higher in RP-ECs than in P-ECs (both, $P<0.0001$ ) (Fig. 2, Panels a and b). Conversely, SOD activity decreased in RP-ECs $(P<0.05)$ compared with P-ECs (Fig. 2, Panel c).

In the presence of $\mathrm{H}_{2} \mathrm{O}_{2}$-induced oxidative stress, Sirt1 and Cat activities were higher in RP-ECs than in P-ECs $(P<0.0001$ and $P<0.05$, respectively) (Fig. 2, Panels a and $b$ ), whereas SOD activity did not change (Fig. 2, Panel c).

These results showed that a CRP induced Sirt1 and Cat activation in both the absence and presence of oxidative stress, suggesting the role of Sirt1 in stimulating the antioxidant response.

\section{Role of Sirt1 and Cat in endothelial cell senescence}

The senescence in endothelial cells (ECs) conditioned with patients' sera was measured. ECs conditioned with sera from patients at the end of a 4-week CRP (RP-ECs) showed a significantly reduced senescence compared to that conditioned with sera from patients before CRP (PECs), in both the absence and presence of induced oxidative stress (both, $P<0.0001$; Fig. 3 ).

To investigate the possible role played by Sirt1 and its molecular target Cat in the modulation of cell senescence, P-ECs and RP-ECs, either exposed or not to oxidative stress, were treated with Sirt1 and Cat pharmacological inhibitors, EX-527 and 3-amino-1,2,4triazole (ATZ) respectively.

As shown in Fig. 3, the inhibition of Sirt1 activity by EX-527 caused an increase of senescence in RP-ECs compared with baseline $(P=0.001)$, but not in P-ECs. Interestingly, in the presence of $\mathrm{H}_{2} \mathrm{O}_{2}$ oxidative stress, EX-527 induced a rise in senescence, in both P-ECs $(P<$ $0.05)$ and RP-ECs $(P=0.001)$. Hence, Sirt1 inhibition abolished the anti-senescent effect of a CRP, suggesting Sirt1 as a modulator of endothelial cell senescence.

Also the inhibition of Cat activity by ATZ resulted in an increased senescence, in both P-ECs and RP-ECs (both, $P<0.0001$ ), compared with baseline. In the presence of $\mathrm{H}_{2} \mathrm{O}_{2}$-induced oxidative stress, ATZ treatment caused a significant increase in the senescence rate in ECs conditioned with RP sera $(P<0.0001)$ compared with basal levels. Of note, senescence become higher in stressed RP-ECs compared with stressed P-ECs when Cat was inhibited $(P=0.001)$. These data suggest that Cat is, at least in part, responsible for the reduced senescence rate observed in ECs conditioned with RP sera.

\section{Discussion}

Most of the studies in both young and older adults have been planned after considering functional and hemodynamic outcomes, without clarifying the effects of a CRP from a molecular point of view.
In this study, we showed an increase of Sirt1 activity in PBMCs alongside an increase of antioxidant capability in serum isolated from patients with HF after 4 weeks of a CRP.

In vivo-in vitro experiments performed in endothelial cells conditioned with patients before and after CRP showed that serum from the rehabilitated patients is able to stimulate Sirt1 activity and the cellular antioxidant defence by increasing activity of the Sirt1 target Cat.

Furthermore, the conditioning of human endothelial cells with serum from rehabilitated patients attenuated senescence in both the absence and presence of oxidative stress induction and such effect was eliminated by the pharmacological inhibition of Sirt1 or Cat activity.

Cellular senescence is a hallmark of ageing and a process in which competent cells are brought into a permanent form of growth arrest. If and how senescence is correlated with age-associated frailty and diseases is still one of the major unanswered questions in ageing physiology and clinical geriatrics [23]. An increase of oxidative stress-induced senescence can be dangerous to endothelial cells, resulting in impairment of endothelial structure and function. Some authors showed that cellular senescence is involved in endothelial dysfunction and atherogenesis, and this was confirmed by a histological study on atherosclerotic human plaques demonstrating morphological features of senescence [24]. As oxidative stress-induced endothelial dysfunction is strictly connected to HF, researching methods to modify this condition is certainly of clinical interest.

The role played by Sirt1 in the regulation of ageing, endothelial homeostasis and cellular senescence is now recognized. Indeed, several studies demonstrated that a $\mathrm{H}_{2} \mathrm{O}_{2}$ treatment caused a reduction of Sirt1 protein expression, and the inhibition of Sirt1 contributed to a $\mathrm{H}_{2} \mathrm{O}_{2}$-induced senescence in endothelial cells $[25,26]$. Furthermore, the Sirt1 target Cat was also shown to be involved in ageing and senescence control [27, 28]. We previously demonstrated that Cat is reduced during ageing [16], and involved in the reduction of endothelial senescence during an aerobic exercise training [20, 21]. Some studies in animal models demonstrated that over-expression of Cat in heart and vessels may have a beneficial impact on HF. In particular, Cat may prevent adverse myocardial remodelling and contribute to the preservation of geometric and functional changes by alleviating stress in the endoplasmic reticulum [18, 29].

Notably, patients enrolled in the present study were HF elderly patients with preserved ejection fraction, a phenotype of HF that is attracting particular attention from both physicians and researchers. Actually, pharmacological trials performed to assess improving of outcome and symptoms, including exercise intolerance, in 


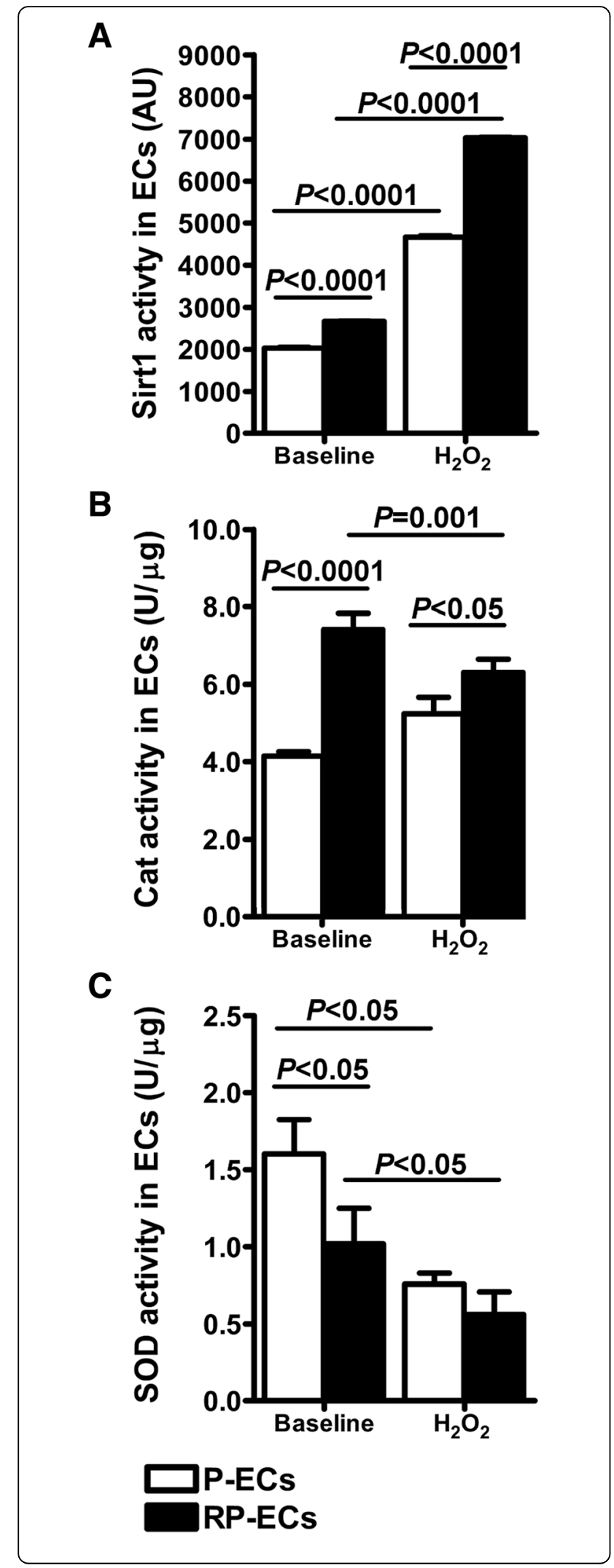

Fig. 2 Sirt1, Cat and SOD activities in conditioned endothelial cells. Sirt1 (a), Cat (b) and SOD (c) activities in endothelial cells (ECS) conditioned with sera from patients before (P) and after (RP) a 4-week well-structured CRP in either the presence or absence of $\mathrm{H}_{2} \mathrm{O}_{2}$-induced oxidative stress

HFpEF patients have been shed light the absence of effective drugs [30, 31].

On the other hand, some recent studies in such patients have suggested that exercise training is a promising therapeutic strategy to improve exercise intolerance [32], increase exercise capacity, as measured objectively using peak oxygen consumption, and ameliorate quality of life and diastolic function, assessed by echocardiography $[6,7,33]$.

Here we showed that, in addition to the improvement of hemodynamic parameters and exercise tolerance (assessed by cardiopulmonary stress test), an exercisebased CRP increases Sirt1 activity and stimulates a systemic antioxidant defence in HFpEF elderly patients and was able to produce antioxidant and anti-senescent effects in endothelial cells mediated, at least in part, by Sirt1 and its target Cat.

\section{Limitations}

A possible limitation of the present study could be the lack of a group of heart failing patients not undergone CR. However, the main outcome was represented by the investigation of the molecular changes occurred before and after a well-structured 4-week rehabilitation

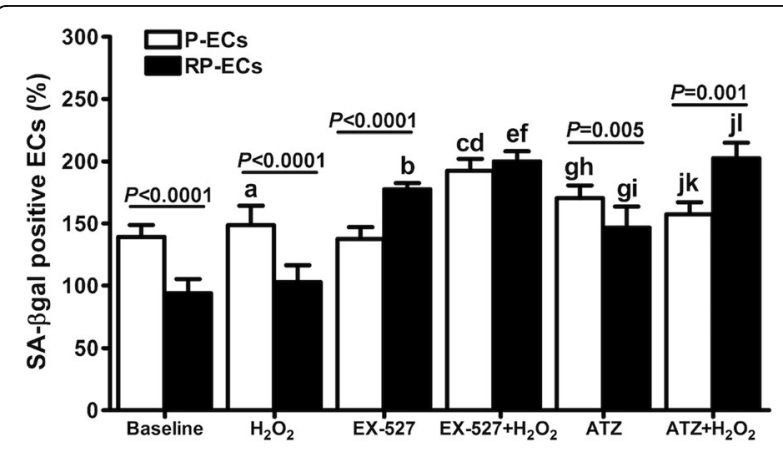

Fig. 3 Effect of the inhibition of Sirt1 and Cat activities on endothelial cell senescence. SA- $\beta$-gal staining of ECs conditioned with sera from HF patients in the presence and absence of oxidative stress. Sirt 1 and Cat activities were inhibited by EX-527 and 3-amino1,2,3-triazole (ATZ), respectively. Senescence values are shown as a percentage of the reference condition (FBS-conditioned $\mathrm{ECS}$ ), which is $100 \%$. a) $P<0.05$ vs baseline; b) $P=0.001$ vs baseline; c) $P<0.05$ vs $\mathrm{H}_{2} \mathrm{O}_{2}$; d) $P=0.01$ vs EX-527; e) $P=0.001$ vs $\mathrm{H}_{2} \mathrm{O}_{2}$; f) $P<0.02$ vs EX-527; g) $P<0.0001$ vs baseline; h) $P<0.01$ vs EX-527;i) $P<0.05$ vs EX-527; j) $P<0.05$ vs ATZ; k) $P<0.02$ vs EX-527 $+\mathrm{H}_{2} \mathrm{O}_{2}$; I) $P<0.0001$ vs $\mathrm{H}_{2} \mathrm{O}_{2}$. (B): a) $P=0.001$ vs baseline; $b) P<0.02$ vs baseline; $c) P<0.005$ vs baseline; d) $P=0.002$ vs $\mathrm{H}_{2} \mathrm{O}_{2}$; e) $P=0.01$ vs EX-527; f) $P<0.01$ vs baseline; g) $P<0.05$ vs EX-527; h) $P<0.05$ vs $\mathrm{H}_{2} \mathrm{O}_{2}$; i) $P=0.002$ vs EX$\left.527+\mathrm{H}_{2} \mathrm{O}_{2} ; \mathrm{j}\right) P=0.001$ vs EX-527 $\left.+\mathrm{H}_{2} \mathrm{O}_{2} ; \mathrm{k}\right) P<0.005$ vs $\mathrm{H}_{2} \mathrm{O}_{2}$ 
program in patients affected by chronic HF, who did not change their clinical characteristics and pharmacological therapy during the study period.

Another drawback is the lack of women in the study population. Actually, we did recruit only three women, and then we decided to exclude them because of the small number. This is in line with the fact that women are less inclined to take part in cardiac rehabilitation programs [34]. Therefore, further studies are necessary to better clarify the molecular effects of $\mathrm{CR}$ also in female patients.

\section{Conclusion}

The ability of exercise training to regulate vascular endothelial function and oxidative stress response is an example of how lifestyle and/or tools such as exercisebased CRPs can complement both clinical and pharmacological means of managing CVDs. In particular, cardiac rehabilitation is a helpful medical practice in which several molecular factors mutually influence each other. The exercise training included in CRPs acts as a non-pharmacological inductor of antioxidant response.

To determine the molecular mechanisms underlying the beneficial effects of CRPs is an essential step in developing a strategy to facilitate the clinical practice of exercise training. Further studies should be addressed to evaluate the possibility of reducing the number and the dosage of drugs in HF patients, including those with preserved ejection fraction by implementing exercise programs, especially in high-risk elderly subjects.

\section{Methods}

\section{Study Design and Population}

A prospective longitudinal observational study was conducted on patients consecutively admitted to the Cardiac Rehabilitation Unit of "San Gennaro dei Poveri" Hospital in Naples, Italy. Patients' information and consent forms were approved by the ethical committee of ASL of Salerno Registry of Observational Studies (RSO) n.10/14.

The study was performed in accordance with the Declaration of Helsinki Seventh Revision (2013) and its amendments. This report adheres to the standards for the reporting of observational trials and was written according to the STROBE guidelines for Observational Studies in Epidemiology - Molecular Epidemiology (STROBE-ME) [35].

Exclusion criteria included unstable angina pectoris, uncompensated HF, complex ventricular arrhythmias, pacemaker implantation and orthopaedic or neurological limitations to exercise.

All enrolled patients underwent a physical examination, collection of demographic and routine blood chemistry tests, chest X-ray, blood pressure measurement, electrocardiographic and echocardiographic examinations, cardiopulmonary stress test and a 6-min walking test with Borg index evaluation. For interval training, low muscle commitment calisthenics and respiratory exercises were performed.

\section{Training Protocol}

The CRP consisted of 30-min sessions of aerobic exercise, 5 days a week. A daily training session comprised a warm-up (10 $\mathrm{min})$, endurance training (15 $\mathrm{min})$ and a cool-down $(5 \mathrm{~min})$ on a cycle ergometer at $50 \%$ of the VO2 max achieved on the cardiopulmonary stress test.

\section{Blood Sample Collection}

Overnight fasting blood samples were obtained from patients before starting and at the end of the CRP. After centrifugation at $1500 \times g$ for $10 \mathrm{~min}$, serum samples were transferred to new tubes and stored at $-80{ }^{\circ} \mathrm{C}$ until analysis. PBMCs were isolated from whole blood by Ficoll-Paque PLUS (GE Healthcare, Munich, Germany), according to manufacturer's procedures.

Samples isolated from patients before CR were indicated as $\mathrm{P}$, while those collected from patients after $\mathrm{CR}$ were designated as RP.

\section{Cell Culture and Treatments}

Human Umbilical Vein Endothelial Cells (HUVECs, ECs) were purchased from Clonetics (Walkersville, MD). The cells were cultured in an endothelial growth medium, containing FBS at a concentration of $2 \%$ and bovine brain extract (with FGF-2 at a concentration of $100-500 \mathrm{pg} / \mathrm{ml}$ ). The cells were subcultured by trypsinization, seeded on cell culture dishes coated with $0.1 \%$ gelatin and growth in an atmosphere of $5 \% \mathrm{CO} 2$ at $37{ }^{\circ} \mathrm{C}$. Pilot experiments to identify the concentration of hydrogen peroxide $\left(\mathrm{H}_{2} \mathrm{O}_{2}=100-750 \mu \mathrm{M}\right)$ that effectively induced a significant decrease in the survival of control cells, were conducted and a concentration of $500 \mu \mathrm{M}$ was chosen. Moreover, we evaluated the effect of oxidative stress $12,24,48$, and $72 \mathrm{~h}$ after a treatment with $500 \mu \mathrm{M}$ $\mathrm{H}_{2} \mathrm{O}_{2}$. Finally, we chose the time of $48 \mathrm{~h}$ as representative of the most relevant change.

Therefore, ECs were seeded and cultured for $48 \mathrm{~h}$ in a medium supplemented with either the patient's serum (10\%) at time 0 (Patient serum-conditioned ECs, P-ECs) and post CR (Rehabilitated Patient serum-conditioned ECs, RP-ECs), or FBS (10\%) as a control and were exposed or not to oxidative stress induced by $500 \mu \mathrm{M}$ $\mathrm{H}_{2} \mathrm{O}_{2}$. Four hours after $\mathrm{H}_{2} \mathrm{O}_{2}$ exposure, the growth medium was replaced with fresh medium containing FBS.

All experiments were performed at a population doubling level (PDL) of 8 to 12 . 


\section{Sirt1 Activity}

Crude nuclear samples were extracted by suspending the cells into $1 \mathrm{~mL}$ of lysis buffer $(10 \mathrm{mM}$ of Tris $\mathrm{HCl}$ at $\mathrm{pH} 7.5,10 \mathrm{mM}$ of $\mathrm{NaCl}, 15 \mathrm{mM}$ of $\mathrm{MgCl}_{2}, 250 \mathrm{mM}$ of sucrose, $0.5 \% \mathrm{NP}-40,0.1 \mathrm{mM}$ of EGTA). Cells were spun through $4 \mathrm{ml}$ of sucrose cushion (30\% sucrose, $10 \mathrm{mM}$ of Tris $\mathrm{HCl}$ at $\mathrm{pH} 7.5,10 \mathrm{mM}$ of $\mathrm{NaCl}, 3 \mathrm{mM}$ of $\mathrm{MgCl}_{2}$ ) at $1300 \times g$ for $10 \mathrm{~min}$ at $4{ }^{\circ} \mathrm{C}$. The isolated nuclei were suspended in $50-100 \mu \mathrm{l}$ of extraction buffer $(50 \mathrm{mM}$ of HEPES $\mathrm{KOH}$ at $\mathrm{pH} 7.5,420 \mathrm{mM}$ of $\mathrm{NaCl}, 0.5 \mathrm{mM}$ of EDTA $\mathrm{Na}_{2}, 0.1 \mathrm{mM}$ of EGTA, $10 \%$ glycerol). After centrifugation at $15,000 \mathrm{rpm}$ for $10 \mathrm{~min}$, the protein concentration of the crude nuclear extract without protease inhibitor was determined by the Bradford method. Sirt1 activity in the nuclei was determined using the CycLex SIRT1/Sir2 Deacetylase Fluorometric Assay Kit (Ina, Nagano, Japan). The reaction was carried out by simultaneously mixing fluorescent-labeled acetylated peptide as substrate and $10 \mu \mathrm{l}$ of the sample, trichostatin A, NAD, and lysyl endopeptidase. The intensity of the fluorescence at $440 \mathrm{~nm}$ was measured $60 \mathrm{~min}$ after the onset of the reaction. Values are reported as relative fluorescence/ $\mu \mathrm{g}$ of protein (AU). All data are the means \pm standard deviation (SD) of three independent experiments.

Catalase and Superoxide Dismutase Antioxidant Activities Catalase (Cat) and superoxide dismutase (SOD) activities were determined using the Catalase Assay Kit and the Superoxide Dismutase Assay Kit (Cayman Chemical, USA). Samples were previously diluted with buffer (1:10 for serum; 1:2 for cell lysate). The values were reported as $U / \mu$ g of protein. All data are the means \pm SD of three independent experiments.

\section{Sirt1 and Catalase Activities Inhibition}

To investigate if Sirt1 or Cat activity influenced changes in the senescence of the conditioned cells either exposed or not to $\mathrm{H}_{2} \mathrm{O}_{2}$, their respective activities were inhibited using EX-527 (Sigma, Milan-Italy) at a concentration of $5 \mu \mathrm{M}$ for $1 \mathrm{~h}$ and 3-amino-1,2,4-triazole (ATZ) (Sigma, Milan-Italy) at a concentration of $10 \mathrm{mM}$ for $3 \mathrm{~h}$.

\section{Senescence-Associated $\beta$-galactosidase (SA- $\beta$-gal) Activity} Cultured cells were washed in PBS and fixed with $2 \%$ formaldehyde and $2 \%$ glutaraldehyde for $10 \mathrm{~min}$ at room temperature. The cells were washed and then incubated at $37{ }^{\circ} \mathrm{C}$ in staining buffer with the following components: $40 \mathrm{mM}$ citric acid/sodium phosphate ( $\mathrm{pH} 6.0$ ), $0.15 \mathrm{M} \mathrm{NaCl}, 2 \mathrm{mM} \mathrm{MgCl}$, $5 \mathrm{mM}$ potassium ferrocyanide, and $1 \mathrm{mg} / \mathrm{mL} \mathrm{X}$-gal (5-bromo-4 chloro-3-indolyl $\beta$ $\mathrm{D}$-galactoside). After $4 \mathrm{~h}$, the SA- $\beta$-gal rate was obtained by counting four random fields per dish and assessing the percentage of SA- $\beta$-gal-positive cells from 100 cells per field. Senescence values are shown as a percentage of the reference condition (FBS-conditioned ECs), which is $100 \%$.

\section{Statistical Analysis}

Continuous variables are expressed as mean \pm SD and compared by paired or unpaired Student's $t$ test (normally distributed variables) or by two or three way ANOVA when appropriate, or as median \pm interquartile range value and compared by the Mann-Whitney $U$ test (not normally distributed). Normality of data distribution was evaluated using the Kolmogorov-Smirnov test. Non-normally distributed continuous variables were converted to their natural log functions. Categorical variables are expressed as a proportion and compared by the $x^{2}$ test, with risk ratios and 95\% confidence intervals quoted.

All data were analysed using SPSS version 19.0 (SPSS, Inc., Chicago, Illinois-USA). Statistical significance was accepted at $P<0.05$.

\section{Abbreviations \\ EX-527: Sirtuin 1 inhibitor; ACCF: American College of Cardiology Foundation; AHA: American Heart Association; ARBs: Angiotensin II Receptor Blockers; ATZ: 3-amino-1,2,4-triazole; AU: Arbitrary unit; BMI: Body mass index; bpm: Beat/minutes; CABG: Coronary artery bypass graft; CAD: Coronary artery disease; Cat: Catalase; COPD: Chronic obstructive pulmonary disease; CRP: Cardiac rehabilitation programme; CVDs: Cardiovascular diseases; DBP: Diastolic blood pressure; ECs: Endothelial cells; EF: Ejection fraction; EGTA: Ethylene glycol tetraacetic acid; ESC: European Society of Cardiology; FBS: Foetal bovine serum; $\mathrm{H}_{2} \mathrm{O}_{2}$ : Oxygen peroxide; $\mathrm{HCl}$ : Hydrogen chloride; HEPES: 4-(2-hydroxyethyl)-1-piperazineethanesulfonic acid; HF: Heart failure; HR: Heart rate; $\mathrm{KOH}$ : Potassium hydroxide; LVEDD: Left ventricle end diastolic diameter; $\mathrm{MgCl}_{2}$ : Magnesium chloride; MI: Myocardial infarction; \\ MnSOD: Manganese-dependent superoxide dismutase; $\mathrm{NaCl}$ : Sodium chloride; $\mathrm{NAD}^{+}$: Nicotinamide adenine dinucleotide coenzyme; NP-40: 4- nonylphenyl-polyethylene glycol; NYHA: New York Heart Association functional classification of heart failure; $\mathrm{O}_{2}$ : Oxygen; P: Patients before cardiac rehabilitation programme; PDL: Population doubling level; P-ECs: Patient serum-conditioned endothelial cells; PTCA: Percutaneous transluminal coronary angioplasty; ROS: Reactive oxygen species; RP: Rehabilitated patients; RP-ECs: Rehabilitated patient serum-conditioned endothelial cells; SA- $\beta$-gal: Senescence-Associated $\beta$-galactosidase; SBP: Systolic blood pressure; Sirt1: Sirtuin 1; SOD: Superoxide dismutase; VO2: Maximal oxygen consumption; X-gal: 5-bromo-4 chloro-3-indolyl $\beta$-D-galactoside.}

\section{Acknowledgements \\ None.}

\section{Funding}

This work was supported by the Department of Medicine, Surgery and Dentistry of University of Salerno [ORSA128783 to A.F.] and the Department of Medicine and Health Sciences of the University of Molise [R-

DIPA_20112013300118CORBI-GR to G.C.].

\section{Availability of data and materials}

The datasets generated and/or analyzed during this study are available from the corresponding author on request.

\section{Authors' contributions}

All authors read and met Immun. Ageing criteria for authorship. Graziamaria Corbi, Valeria Conti, Nicola Ferrara and Amelia Filippelli conceived and designed the experiments. Giusy Russomanno, Valentina Manzo, Giuseppe Rengo and Albino Carrizzo performed the experiments. Salvatore Latte and Maria Consiglia Calabrese contributed with acquisition of clinical data and 
human samples. Graziamaria Corbi, Valeria Conti and Giusy Russomanno performed the analysis and interpretation of the data and wrote the paper. Nicola Ferrara, Annibale A. Puca, Ramaroson Andriantsitohaina, Walter Filippelli, Carmine Vecchione, Amelia Filippelli critically revised the paper. All authors read and approved the final paper.

\section{Competing interests}

The authors have no conflicts of interest to report.

\section{Consent for publication}

All authors have read and agreed to its content and are accountable for all aspects of the accuracy and integrity of the manuscript in accordance with ICMJE criteria

\section{Ethics approval and consent to participate}

Patients' information and consent forms were approved by the ethical committee of ASL of Salerno Registry of Observational Studies (RSO) n.10/14.

\section{Author details}

'Department of Medicine, Surgery and Dentistry, University of Salerno, Via S. Allende 43, 84081 Baronissi, Italy. ${ }^{2}$ Department of Medicine and Health Sciences, University of Molise, Campobasso, Italy. ${ }^{3}$ Department of Translational Medical Sciences, Federico II University of Naples, Naples, Italy. ${ }^{4}$ Salvatore Maugeri Foundation, IRCCS, Scientific Institute of Telese Terme, Benevento, Italy. ${ }^{5}$ RCCS MultiMedica, Milan, Italy. ${ }^{6}$ Cardiac Rehabilitation Unit of "San Gennaro dei Poveri" Hospital, Naples, Italy. "Vascular Physiopathology Unit, IRCCS INM Neuromed, Pozzilli, Italy. ${ }^{8}$ INSERM U1063, Stress Oxydant et Pathologies Métaboliques, Institut de Biologie en Santé, Université d'Angers, Angers, France. ${ }^{9}$ Department of Institutional Study and Territorial Systems, University of Naples "Parthenope", Naples, Italy.

Received: 12 November 2016 Accepted: 24 February 2017 Published online: 16 March 2017

\section{References}

1. Mozaffarian D, Benjamin EJ, Go AS, Arnett DK, Blaha MJ, Cushman M, et al. Heart disease and stroke statistics-2015 update: a report from the American Heart Association. Circulation. 2015;131:e29-322.

2. Lawler PR, Filion KB, Eisenberg MJ. Efficacy of exercise-based cardiac rehabilitation post-myocardial infarction: a systematic review and metaanalysis of randomized controlled trials. Am Heart J. 2011;162:571-84. e2.

3. Schutzer KA, Graves BS. Barriers and motivations to exercise in older adults. Prev Med. 2004;39:1056-61.

4. Rengo G, Leosco D, Zincarelli C, Marchese M, Corbi G, Liccardo D, et al. Adrenal GRK2 lowering is an underlying mechanism for the beneficial sympathetic effects of exercise training in heart failure. Am J Physiol Heart Circ Physiol. 2010;298:H2032-2038.

5. Leosco D, Rengo G, laccarino G, Golino L, Marchese M, Fortunato F, et al. Exercise promotes angiogenesis and improves beta-adrenergic receptor signalling in the post-ischaemic failing rat heart. Cardiovasc Res. 2008;78:385-94.

6. Nolte $K$, Herrmann-Lingen $C$, Wachter $R$, Gelbrich $G$, Düngen $H-D$, Duvinage $A$, et al. Effects of exercise training on different quality of life dimensions in heart failure with preserved ejection fraction: the Ex-DHF-P trial. Eur J Prev Cardiol. 2015:22:582-93.

7. Ismail H, MCFarlane JR, Nojoumian AH, Dieberg G, Smart NA. Clinical outcomes and cardiovascular responses to different exercise training intensities in patients with heart failure: a systematic review and metaanalysis. JACC Heart Fail. 2013;1:514-22.

8. Corbi G, Conti V, Russomanno G, Rengo G, Vitulli P, Ciccarelli AL, et al. Is physical activity able to modify oxidative damage in cardiovascular aging? Oxid Med Cell Longev. 2012;2012:728547.

9. Meyer P, Gayda M, Juneau M, Nigam A. High-intensity aerobic interval exercise in chronic heart failure. Curr Heart Fail Rep. 2013;10:130-8.

10. Tanno M, Kuno A, Horio Y, Miura T. Emerging beneficial roles of sirtuins in heart failure. Basic Res Cardiol. 2012;107:273.

11. Corbi G, Conti V, Russomanno G, Longobardi G, Furgi G, Filippelli A, et al. Adrenergic signaling and oxidative stress: a role for sirtuins? Front Physiol. 2013;4:324.

12. Yu W, Fu Y-C, Chen C-J, Wang X, Wang W. SIRT1: a novel target to prevent atherosclerosis. J Cell Biochem. 2009;108:10-3.
13. Rahman M, Halade GV, Bhattacharya A, Fernandes G. The fat-1 transgene in mice increases antioxidant potential, reduces pro-inflammatory cytokine levels, and enhances PPAR-gamma and SIRT-1 expression on a calorie restricted diet. Oxid Med Cell Longev. 2009;2:307-16.

14. Conti V, Corbi G, Simeon V, Russomanno G, Manzo V, Ferrara N, et al. Aging related changes in oxidative stress response of human endothelial cells. Aging Clin Exp Res. 2015;27:547-53.

15. Lu T-M, Tsai J-Y, Chen Y-C, Huang C-Y, Hsu H-L, Weng C-F, et al. Downregulation of Sirt1 as aging change in advanced heart failure. J Biomed Sci. 2014;21:57.

16. Ferrara N, Rinaldi B, Corbi G, Conti V, Stiuso P, Boccuti S, et al. Exercise training promotes SIRT1 activity in aged rats. Rejuvenation Res. 2008:11:139-50.

17. Leopold JA, Loscalzo J. Oxidative enzymopathies and vascular disease Arterioscler Thromb Vasc Biol. 2005:25:1332-40.

18. Qin F, Lennon-Edwards S, Lancel S, Biolo A, Siwik DA, Pimentel DR, et al. Cardiac-specific overexpression of catalase identifies hydrogen peroxidedependent and -independent phases of myocardial remodeling and prevents the progression to overt heart failure in G(alpha)q-overexpressing transgenic mice. Circ Heart Fail. 2010;3:306-13.

19. Conti V, Forte $M$, Corbi G, Russomanno G, Formisano L, Landolfi A, Izzo V, Filippelli A, Vecchione C, Carrizzo A. Sirtuins: Possible Clinical Implications in Cardio and Cerebrovascular Diseases. Curr Drug Targets. 2017;18(4):473-84.

20. Conti V, Corbi G, Russomanno G, Simeon V, Ferrara N, Filippelli W, et al. Oxidative stress effects on endothelial cells treated with different athletes' sera. Med Sci Sports Exerc. 2012;44:39-49.

21. Conti V, Russomanno G, Corbi G, Guerra G, Grasso C, Filippelli W, et al. Aerobic training workload affects human endothelial cells redox homeostasis. Med Sci Sports Exerc. 2013;45:644-53.

22. Ponikowski P, Voors AA, Anker SD, Bueno H, Cleland JGF, Coats AJS, et al. 2016 ESC Guidelines for the diagnosis and treatment of acute and chronic heart failure: The Task Force for the diagnosis and treatment of acute and chronic heart failure of the European Society of Cardiology (ESC)Developed with the special contribution of the Heart Failure Association (HFA) of the ESC. Eur Heart J. 2016;37:2129-200.

23. Campisi J. Cellular senescence: putting the paradoxes in perspective. Curr Opin Genet Dev. 2011;21:107-12.

24. Minamino T, Komuro I. Vascular cell senescence: contribution to atherosclerosis. Circ Res. 2007;100:15-26.

25. Ota H, Eto M, Kano MR, Ogawa S, lijima K, Akishita M, et al. Cilostazol inhibits oxidative stress-induced premature senescence via upregulation of Sirt1 in human endothelial cells. Arterioscler Thromb Vasc Biol. 2008:28:1634-9.

26. Song Z, Liu Y, Hao B, Yu S, Zhang H, Liu D, et al. Ginsenoside Rb1 prevents H2O2-induced HUVEC senescence by stimulating sirtuin-1 pathway. PLoS One. 2014;9:e112699.

27. Ota H, Eto M, Kano MR, Kahyo T, Setou M, Ogawa S, et al. Induction of endothelial nitric oxide synthase, SIRT1, and catalase by statins inhibits endothelial senescence through the Akt pathway. Arterioscler Thromb Vasc Biol. 2010;30:2205-11.

28. Schriner SE, Linford NJ, Martin GM, Treuting P, Ogburn CE, Emond M, et al. Extension of murine life span by overexpression of catalase targeted to mitochondria. Science. 2005:308:1909-11.

29. Ge W, Ge W, Zhang Y, Han X, Ren J. Cardiac-specific overexpression of catalase attenuates paraquat-induced myocardial geometric and contractile alteration: role of ER stress. Free Radic Biol Med. 2010;49:2068-77.

30. Massie BM, Carson PE, McMurray JJ, Komajda M, McKelvie R, Zile MR, Anderson S, Donovan M, Iverson E, Staiger C, Ptaszynska A. I-PRESERVE Investigators. Irbesartan in patients with heart failure and preserved ejection fraction. N Engl J Med. 2008;359:2456-67.

31. Kitzman DW, Hundley WG, Brubaker PH, Morgan TM, Moore JB, Stewart KP, Little WC. A randomized double-blind trial of enalapril in older patients with heart failure and preserved ejection fraction: effects on exercise tolerance and arterial distensibility. Circ Heart Fail. 2010;3:477-85.

32. Upadhya B, Haykowsky MJ, Eggebeen J, Kitzman DW. Exercise intolerance in heart failure with preserved ejection fraction: more than a heart problem. J Geriatr Cardiol. 2015;12:294-304.

33. Ponikowski P, Voors AA, Anker SD, Bueno H, Cleland JGF, Coats AJS, et al. 2016 ESC Guidelines for the diagnosis and treatment of acute and chronic heart failure: The Task Force for the diagnosis and treatment of acute and chronic heart failure of the European Society of Cardiology (ESC). 
Developed with the special contribution of the Heart Failure Association (HFA) of the ESC. Eur J Heart Fail. 2016;18:891-975.

34. Grace SL, Racco C, Chessex C, Rivera T, Oh P. A narrative review on women and cardiac rehabilitation: program adherence and preferences for alternative models of care. Maturitas. 2010;67(3):203-8. doi:10.1016/j. maturitas.2010.07.001.

35. Gallo V, Egger M, McCormack V, Farmer PB, loannidis JPA, Kirsch-Volders M, et al. STrengthening the Reporting of OBservational studies in Epidemiology-Molecular Epidemiology (STROBE-ME): an extension of the STROBE Statement. PLoS Med. 2011;8:e1001117.

Submit your next manuscript to BioMed Central and we will help you at every step:

- We accept pre-submission inquiries

- Our selector tool helps you to find the most relevant journal

- We provide round the clock customer support

- Convenient online submission

- Thorough peer review

- Inclusion in PubMed and all major indexing services

- Maximum visibility for your research

Submit your manuscript at www.biomedcentral.com/submit 